\title{
Conf-950261--2
}

UCRL-JC-119050

PREPRINT

\section{Halbach Array Motor/Generators A Novel Generalized Electric Machine}

B. T. Merritt, R. F. Post, G. R. Dreifuerst, D. A. Bender

This paper was prepared for submittal to

Halbach Festschrift Symposium, Berkeley, California

February 3, 1995

October 28, 1994

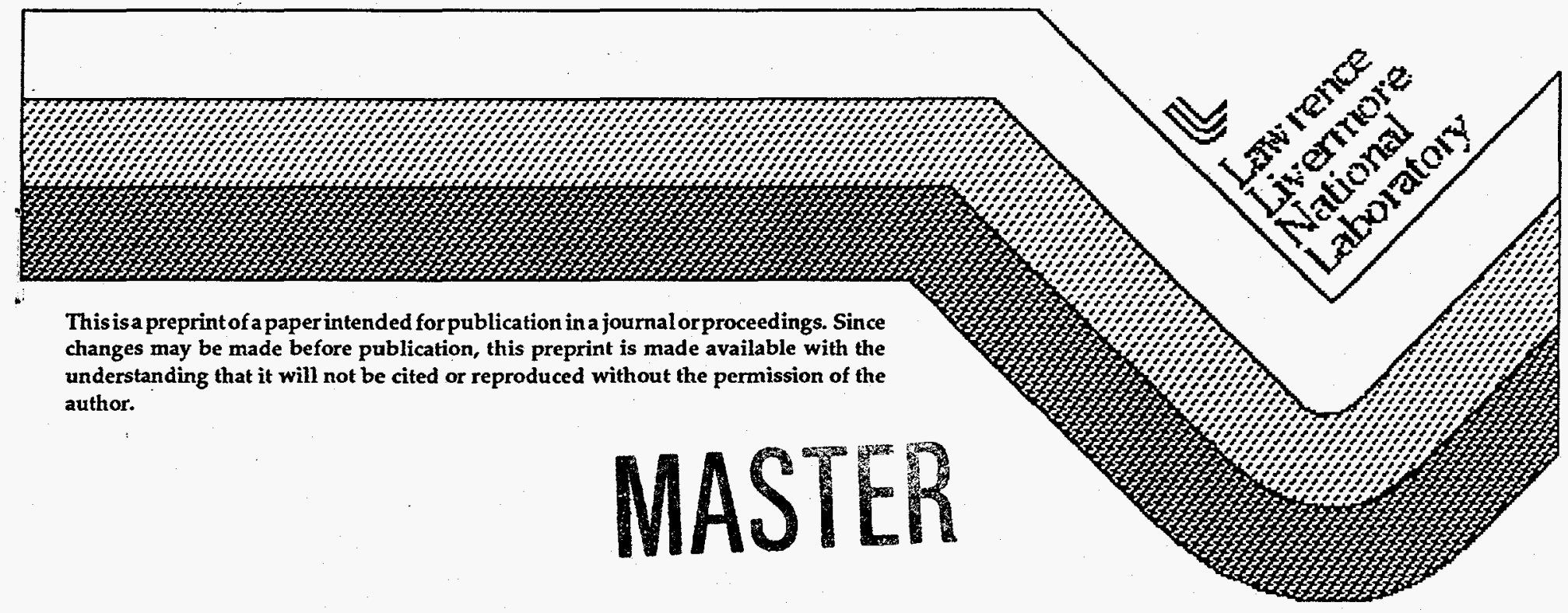

MOTBBUTON OF THS DOUMW IS UMLMTED $W w$ 


\section{DISCLAIMER}

This report was prepared as an account of work sponsored by an agency of the United States Government. Neither the United States Government nor any agency thereof, nor any of their employees, make any warranty, express or implied, or assumes any legal liability or responsibility for the accuracy, completeness, or usefulness of any information, apparatus, product, or process disclosed, or represents that its use would not infringe privately owned rights. Reference herein to any specific commercial product, process, or service by trade name, trademark, manufacturer, or otherwise does not necessarily constitute or imply its endorsement, recommendation, or favoring by the United States Government or any agency thereof. The views and opinions of authors expressed herein do not necessarily state or reflect those of the United States Government or any agency thereof. 


\section{DISCLAIMER}

Portions of this document may be illegible in electronic image products. Images are produced from the best available original document. 


\title{
Halbach Array Motor/Generators - A Novel Generalized Electric Machine
}

\author{
B. T. Merritt, R. F. Post, G. R. Dreifuerst, D. A. Bender \\ Lawrence Livermore National Laboratory
}

\section{Introduction}

For many years Klaus Halbach has been investigating novel designs for permanent magnet arrays, using advanced analytical approaches and employing a keen insight into such systems. One of his motivations for this research was to find more efficient means for the utilization of permanent magnets for use in particle accelerators and in the control of particle beams. As a result of his pioneering work, high power free-electron laser systems, such as the ones built at the Lawrence Livermore Laboratory, became feasible, and his arrays have been incorporated into other particle-focusing systems of various types. This paper reports another, quite different, application of Klaus' work, in the design of high power, high efficiency, electric generators and motors. When tested, these motor/generator systems display some rather remarkable properties. Their success derives from the special properties which these arrays, which we choose to call "Halbach arrays", possess.

In August 1979, K. Halbach submitted a paper ${ }^{1}$ entitled "Design of Permanent Multipole Magnets with Oriented Rare Earth Cobalt Material." In this paper, he presented a novel method of generating multipole magnetic fields using non-intuitive geometrical arrangements of permanent magnets. In subsequent publications $2-4$, he further defined these concepts. Of particular interest to one of the authors (RFP) was the special magnet array that generated a uniform dipole field. In 1990 Post proposed the construction of an electric machine (a motor/generator) using a dipole field based on $\mathrm{K}$. Halbach's array of permanent magnets. He further proposed that such a system should be employed as an integral part of "an electromechanical battery" (EMB), i.e., a modular flywheel system to be used as a device for storing electrical energy, as an alternative to the electrochemical storage battery.

This paper reviews Halbach's theory for the generation of a dipole field using an array of permanent magnet bars, presents a simple analysis of a family of novel "ironless" electric machines designed using the dipole Halbach array, and describes the results obtained when they were tested in the laboratory. 


\section{The Dipole Array}

Although electric machines can be constructed using multipole fields based on the techniques introduced by $\mathrm{K}$. Halbach, the dipole field offers some unique advantages for the construction of a high speed electric machine. Figure 1 shows an end view of a dipole Halbach array. Shown in the figure are the directions of magnetization of the bars and one quadrant of the computed lines of force produced by the array. Note the highly uniform field inside the array, and the near-cancellation of the field outside the array.

Except near the ends of the array, or very near its inner surface, the dipole field inside a such an array is given by the expression derived by Halbach:

$$
B_{0}=B_{r} C_{N} \ln \left(r_{2} / r_{1}\right)
$$

with $r_{1}$ and $r_{2}$ indicating the inside and outside radius of the magnet array, respectively, and $\mathrm{B}_{\mathrm{r}}$ representing the remanent field of the permanent magnet material. If $M$ is the number of segments in the magnet,

$$
\text { * } C_{\mathrm{N}}=\operatorname{Sin}(2 \pi / \mathrm{M}) /(2 \pi / \mathrm{M})
$$

giving $C_{N}=0.90$ for $M=8$ and $C_{N}=0.97$ for $M=16$. These equations are derived in reference 2 .

When Equations (1) and (2) are compared with a computer code using the correct remanent field of the magnets, the code results agree within a percent or two of the analytical result. Arrays constructed using the high-field material, $\mathrm{NdFeB}$, have measured fields that are found to be within a few percent of the predicted values.

Figure 2 is a field plot of a dipole field constructed using an eight segment Halbach array. The permanent magnets used for this dipole were ceramic; the field values measured are uniform to about three percent. The values for the field strength are those measured inside the Halbach array cylinder; the field decreases somewhat near the ends of the magnet array. See references for a detailed discussion and calculation of this decrease. 


\section{Construction of the Generalized Motor/Generator}

The design of a motor/generator using a dipole Halbach array is simplicity itself. Furthermore, since there are no iron laminations used in the design the theoretical prediction of the properties of such motor/generators is equally simple.

In order to construct a motor/generator, all that is required is to insert a single or multi-phase winding down the axis of the dipole field and provide relative motion between the field and the winding(s). Relative rotation then generates an EMF in the winding that is linearly related to the product of the rotation speed and the amount of flux intercepted by the winding. In the case of a generator, the work performed in sustaining the relative motion between the windings and the field is transformed directly into ac electrical energy that flows out of the machine. Conversely, ac electrical energy flowing into the winding, if at the proper frequency and phase, is transformed into mechanical work that causes a relative motion between the windings and the field. At this point it is important to note that only a relative motion between the windings and the field is required; electric machines having either the windings or the Halbach array in motion could be constructed. There are advantages to each type depending upon the application.

Figure 3 shows a three phase winding inserted into the Halbach array field. Single phase or higher phase number machines can also be constructed. This paper concentrates on the three phase machine in which the dipole Halbach array surrounding the winding is rotating. This configuration has been employed by us in the application for which it was first proposed, electromechanical batteries (flywheel energy storage modules).

In order to calculate the induced EMF, consider first the simple case of a single turn winding. Assume for the moment that the winding is stationary and that the Halbach field, due to the relative motion, is given by

$$
B(t)=B_{0} \operatorname{Cos}(\omega t)
$$

Owing to the linearity of the system the induced EMF is given exactly by

$$
V(t)=d \Phi / d t,
$$

where $\Phi$ is simply given by the product of the dipole magnetic field strength and the area, A, intercepted by the windings (with a small correction, usually of order 10 to 20 percent, depending on the relative length of the windings 
and the magnets) for the fall-off of the field strength near the ends of the magnet array.

This expression then reduces to

$$
V(t)=B_{0} A \omega \operatorname{Sin}(\omega t)
$$

where $A$ is the area of the winding, i.e., its length multiplied by its transverse width, and $w$ is the rotational velocity in radians per second.

For the case of multiple turns per winding, the voltage becomes

$$
V(t)=K N B_{0} \omega \operatorname{Sin}(\omega t)
$$

where $\mathrm{N}$ is the number of turns and $\mathrm{K}$ is a geometric constant, close to unity, that accounts for the fact that the winding now has some physical width that spans an arc along the circumference of the cylinder upon which the windings are attached. This constant is readily calculated; it accounts for the relative decrease in magnetic field since a particular turn is no longer exactly aligned with the reference direction for the magnetic field. For such a turn the correction factor is equal to $\cos \beta$, where $\beta$ is the angle between the reference for the magnetic field, usually zero, and the angle at which a particular turn resides. For a distributed winding with angular spread $\alpha$ (i.e. its two sides intercept a fraction $2 \alpha / 2 \pi$ of the circumference), upon performing the average over this spread the factor $\mathrm{K}$ is given by the following expression:

$$
K=2 \operatorname{Sin}(\alpha / 2) / \alpha
$$

If $\alpha=30^{\circ}$, for example, $\mathrm{K}=0.989$.

Figure 4 is a schematic of the electric machine. It is to be interpreted on a per phase basis for machines having multiple phases. Note that this representation contains only linear elements, and also note that the value of magnetic field $\mathrm{B}_{0}$, that is to be used in calculating the induced voltage, is that of the Halbach array. In this ironless system, and for all feasible values of the winding currents, there is no "back reaction" between the stator windings and the inducing magnetic field. There is, of course, an effect of the winding inductance on the output voltage as well as the usual resistive drop. However, since the system is ironless, inductances are low, and with good design, the resistive drops are also low. As will be shown, not only is the 
power output very high, but the efficiency is also typically much higher than that of an iron-core machine of comparable physical size.

The equations for torque are also straightforward. Consider again the case of a single turn winding and only one phase. The vector force on a conductor is given by

$$
\mathbf{F}=2 \mathrm{I}(\hat{\mathrm{L}} \times \mathrm{B})
$$

where $I$ is the current in the conductor, $L$ is the vector path along the conductor and $\mathrm{B}$ is the vector magnetic field. The torque is then

$$
T=r \times F ，
$$

where $\mathbf{r}$ is the radius.

For a single turn rectangular winding with longitudinal length $L$, in a dipole field, the magnitude of the peak torque becomes

$$
\mathrm{T}=2 \mathrm{rLIB}_{0}
$$

and for the multiple turn case, the peak torque becomes

$$
\mathrm{T}=2 \mathrm{rLIB} \mathrm{B}_{0} \mathrm{KN} \text {, }
$$

where $\mathrm{K}$ is the geometric factor as before and $\mathrm{N}$ is the number of turns.

Owing to the complete linearity of this ironless system these simple equations form an adequate basis for the design of motor/generator systems employing a dipole Halbach array. We will later discuss the circuit-related factors that must be employed in order to calculate the output power and the efficiency of such generators. Again, as noted, the absence of iron in the magnetic circuit means that only simple air-core inductances and winding resistances must be taken into account to calculate these quantities.

If the rms output voltage of each of the 3-phase windings is $V_{0}$, the winding inductance is $L_{0}$, and its resistance is $R_{0}$, then the output power of each phase into a resistive load with resistance $R_{L}$ is given by the equation:

$$
P=\frac{\left(V_{0}\right)^{2}}{R_{L}}\left\{\frac{1}{\left[1+\left(R_{0} / R_{L}\right)\right]^{2}+\left(\omega L_{0} / R_{L}\right)^{2}}\right\}, \text { Watts/phase }
$$


The efficiency is then given by the expression:

$$
\eta=\frac{R_{L}}{R_{0}+R_{L}}
$$

\section{Advantages}

A fundamental advantage of a machine of this type has already been mentioned. That is, the fact that the machine can be constructed without the use of magnetic material other than the permanent magnets. There is no need for laminations or back iron. This has two major advantages. First the conventional core loss and eddy current loss in the laminations or back iron does not exist. The only loss in the machine will be losses in the windings, The second advantage is since there is no back iron or laminations required, the machine is inherently lightweight.

The uniform field also results in several important advantages. Since the field is truly uniform, the machine design is no longer constrained by the airgap size. This offers the opportunity to solve other system issues. For example, in the modular EMB application, it allows a vacuum barrier to be placed between the windings and the Halbach array without appreciable degradation of machine performance.

The field uniformity of the dipole Halbach array has another, very important result for the extraction of very high peak powers over short time scales. Conventional generators employing narrow gaps and iron laminations have a problem that is not encountered here. In such systems, where the drag torque caused by the power is a function of the gap spacing, there can exist a strong tendency to drive the rotating system into so-called "whirl" instability. Contrast this with our situation. Since the field is uniform, the torque is not a function of the displacement of the windings relative to the field (the windings only "know" that they are in a uniform rotating field, origin "unknown"). Thus the potential, from this source, for whirl instability does not exist.

As mentioned earlier, the major loss mechanism in this type of machine is the losses in the windings. These losses can be minimized by increasing the amount of copper in the windings. There is a trade-off between the efficiency of the machine and the size of the winding. There is also a potential loss due to eddy currents in the conductors; since the field is always present, eddy currents will be induced in the conductors due to the relative 
motion between the windings and the field. This latter loss is easily controlled to low levels, i.e. a few watts, by the use of Litz wire.

\section{Motor Types}

Halbach array electric machines could be constructed in a conventional manner, whereby the Halbach array is stationary and the windings rotate within the array. As we have mentioned for use in our modular EMBs, the Halbach array machine has been constructed "inside out", with the windings stationary and the magnet array rotating around the windings. There are advantages to both types of construction; specific applications drive the choice.

In a conventional machine configuration, the Halbach array machine would look physically much like a dc machine; that is, the armature is on the rotor and the field is stationary. Such a machine could be operated as either a $\mathrm{dc}$ or ac device. If one uses slip rings to bring out the windings, the machine could be characterized as an ac machine. However, if now an electronic commutation circuit is added, the Halbach array machine can be characterized as a dc machine. If one desired, one could use a mechanical commutator as well.

The inherent advantage of this construction technique is that the inertia of the rotor is dominated solely by the mass of the windings since there are no laminations required. One can therefor envision the construction of a very high speed low inertia machine.

If the machine is configured, as we have done, in an "inside-out" geometry, the windings are stationary and readily accessible. Again, the machine can be characterized as either an ac or dc machine. The primary advantage of the inside-out construction is that it is readily adapted to evacuated systems (such as in our EMB) since there is no need for slip rings.

\section{Our specific application, the EMB}

As noted earlier, the impetus for the development of this new type of electric machine was our application to modular electromechanical batteries. $5,6,7$ The EMB battery consists of a high-speed flywheel with an integral motor/generator suspended on magnetic bearings and in an evacuated housing. For practical use, a set of power electronics is coupled to this module. The flywheel and its motor/generator is a means for energy storage and extraction; the power electronics conditions electrical energy, both for 
adding energy to and extracting energy from the flywheel. Since the goal is to mimic a battery, the input/output voltage to the power electronics is typically dc, although ac-based systems can also be contemplated, using so-called cycloconverters, or the multi-phase devices called matrix converters. A sketch of the electromechanical battery is given in Figure 5.

As can be seen in Figure 5, the Halbach array machine is an integral part of the flywheel construction. The array of magnets is designed into the rotor; the mass of the magnets is used advantageously to keep the composite material in compression. The advantages listed above are also utilized in this design. The outer diameter of the stator windings are typically a centimeter smaller than the inner diameter of the Halbach array magnets. In this space, a thin vacuum barrier is placed, still allowing for a substantial clearance between the array and the barrier. This clearance simplifies the design of the bearing/suspension system, which need not constrain the radial displacements of the rotor assembly to the fraction-of-a-millimeter tolerances that would be required in a conventional iron-core machine.

We conclude this section with a summary of the design parameters of one of the modular EMBs that we have constructed.

\section{Table 1 Electromechanical Battery Parameters}

\begin{tabular}{|c|c|}
\hline Useful Energy ( $100 \%$ to $50 \%$ speed) & $0.6 \mathrm{~kW}-\mathrm{hr}$ \\
\hline Max Speed & $84,000 \mathrm{RPM}$ \\
\hline Peak Power & $50 \mathrm{~kW}$ \\
\hline Open Circuit Voltage & $151 \mathrm{Vrms} /$ phase at max speed \\
\hline Halbach Array Length & $18 \mathrm{~cm}$ \\
\hline Halbach Array Outer Diameter & $10.5 \mathrm{~cm}$ \\
\hline Halbach Array Inner Diameter & $7 \mathrm{~cm}$ \\
\hline Winding - Turns/phase & 6 \\
\hline Conductor size & 1700 strand, $\# 40$ Litz \\
\hline aductance per phase & 7.4 microhenries \\
\hline istance per phase & 10.8 milliohms \\
\hline \multicolumn{2}{|c|}{$\begin{array}{l}\text { Using these data and equations (12) and (13) we can calculate the power } \\
\text { it and the efficiency of this generator. We assume that the power from } \\
\text { of the three phases is summed independently in calculating the output } \\
r \text {, and we ignore the eddy current losses in the Litz wire (it is of order } \\
\text { two Watts). In the table below the power and efficiency are calculated } \\
\text { unction of the load resistance (the same for each of the three phases) and } \\
\text { speed ( } 84,000 \mathrm{RPM}) \text {. As will be seen at or below the design power the } \\
\text { mcies are very high. }\end{array}$} \\
\hline
\end{tabular}


Table 2. Generator Efficiency Versus Output Power

$\begin{array}{lll}\begin{array}{l}\mathbf{R}_{\mathbf{L}} \\ \text { (Ohms) }\end{array} & \begin{array}{l}\text { Power } \\ (\mathrm{kW})\end{array} & \text { Efficien } \\ 3.0 & 23 & 0.9964 \\ 2.5 & 27 & 0.9957 \\ 2.0 & 34 & 0.9946 \\ 1.5 & 45 & 0.9929 \\ 1.0 & 66 & 0.9893\end{array}$

\section{Drive Circuitry}

Although the Halbach array machine can be operated as either an ac or dc machine, there are advantages to operate it as a dc machine. The primary advantage is that the drive circuitry can use a simple $120^{\circ}$ gating waveforms. Normally, in an ac machine, elaborate PWM techniques are used to generate sinusoidal waveforms in order to minimize harmonics that can cause losses in the laminations. Since the Halbach array machine does not have laminations this is no longer of concern. The resulting drive can then be greatly simplified, since in this case a simple rectangular wave is very nearly as efficient in driving the rotor as would be a pure sine wave.

In our specific application, energy is transferred into and out of the flywheel on a routine basis. In order to add energy to the flywheel, the power electronics treat the Halbach array machine as an electronically commutated $\mathrm{dc}$ motor, as the voltage is raised across the motor, the speed of the motor increases. A schematic of the power electronics for spin-up is given in Figure 6 . The electronic commutation is provided by six IGBTs connected to the three phases of the machine. The dc voltage to the commutating bridge is controlled by the "buck" regulator formed by one IGBT, an inductor, and diode. The IGBTs in the commutation bridge also serve as an overcurrent protection scheme. The lower IGBT in each leg can be pulse width modulated to control the current in the windings. The switching frequency for this modulation is about $20 \mathrm{kHz}$, while the electronic commutation needs to accommodate frequencies up to $1400 \mathrm{~Hz}$ to achieve 84,000 RPM operation. This protection is not for the windings but for the power semiconductors.

A schematic for extraction of power from the flywheel is given in Figure 7. The three phases of the windings are connected to a six pulse diode bridge to convert the ac waveforms to $d c$. Since the speed changes a factor of two while energy is being extracted the dc voltage will also decrease by a factor 
of two. To accommodate this change in dc bus voltage a "boost" regulator is added. The boost circuit provides for a constant output voltage by changing the IGBT duty cycle.

Since IGBTs are normally packaged with an antiparallel diode, the spin-up and extraction circuit can be combined to one as shown in Figure 8. This circuit works best when the energy is added to the flywheel on the same time scale as it is extracted. Figure 8 is an example of circuit that creates a true electromechanical battery; the flywheel-power electronics system mimics a dc battery. For those applications where energy compression is important, that is, the energy is added/extracted on a much longer time scale than it is extracted/added, there is a cost advantage to separate the spin-up and extraction functions in order to minimize the cost of the power semiconductors.

\section{Performance}

Although the flywheel system has not yet been operated at full speed, the fundamental characteristics of the Halbach array electric machine have been verified.

\section{AC Operation}

Figure 9a is a typical open circuit voltage waveform; the purity of the sinusoid is given in Figure $9 \mathrm{~b}$ which shows that the highest amplitude harmonic is less than a factor of 300 than the fundamental (i.e., more than $\mathbf{5 0}$ decibels lower).

\section{Extraction of High Power}

Using the flywheel with an integral Halbach array machine, over 50 $\mathrm{kW}$ of power (into a load that was not impedance-matched for maximum power) in about one second bursts have been successfully extracted. During this high peak power, the machine did not exhibit any indications of whirl instability. To perform this extraction, the windings were connected to a six pulse diode bridge that was then switched into a resistive load. Figure 10 shows typical bridge voltage and current during extraction. The peak power that has been extracted to date was $65 \mathrm{~kW}$ for about one second.

DC Operation

The flywheel with an integral Halbach array machine has been routinely operated at 40,000 RPM. During the spin-up, a single phase version 
of the power electronics as shown in Figure 6 was used. Figure 11 is a plot of speed versus dc bus voltage. The dc bus voltage is always slightly higher than the emf to ensure power flow into the motor during spin-up. This operation is typical of a dc motor with constant excitation.

*This work was performed under the auspices of the U.S. Department of Energy by Lawrence Livermore National Laboratory under contract No. W-7405-ENG-48. 
References

1. K. Halbach, "Design of Permanent Multipole Magnets with Oriented Rare Earth Cobalt Material", Nuclear Instruments and Methods, 169, pp. 1-10, (1980).

2. K. Halbach, "Physical and Optical Properties of Rare Earth Cobalt Magnets," Nuclear Instruments and Methods, 187, pp. 109-117, (1981).

3. K. Halbach, "Perturbation Effects in Segmented Rare Earth Cobalt Multipole Magnets," Nuclear Instruments and Methods, 198, pp. 213-215, (1982).

4. K. Halbach, Specialty Magnets, Lawrence Berkeley Laboratory, LBL - 21945, (1986).

5. R. F. Post, T. K. Fowler, and S. F. Post, A High Efficiency Electromechanical Battery, Lawrence Livermore National Laboratory, UCRL-JC-110861, (1992).

6. R. F. Post, D. E. Baldwin, D. A. Bender and T. K. Fowler, Electromechanical Battery Research and Development ant the Lawrence Livermore National Laboratory, Lawrence Livermore National Laboratory, UCRL-JC-113905, (1993).

7. R F. Post, D. A. Bender and B. T. Merritt, "Electromechanical Battery Program at the Lawrence Livermore National Laboratory," IECEC, Monterey, CA, August 1994. 

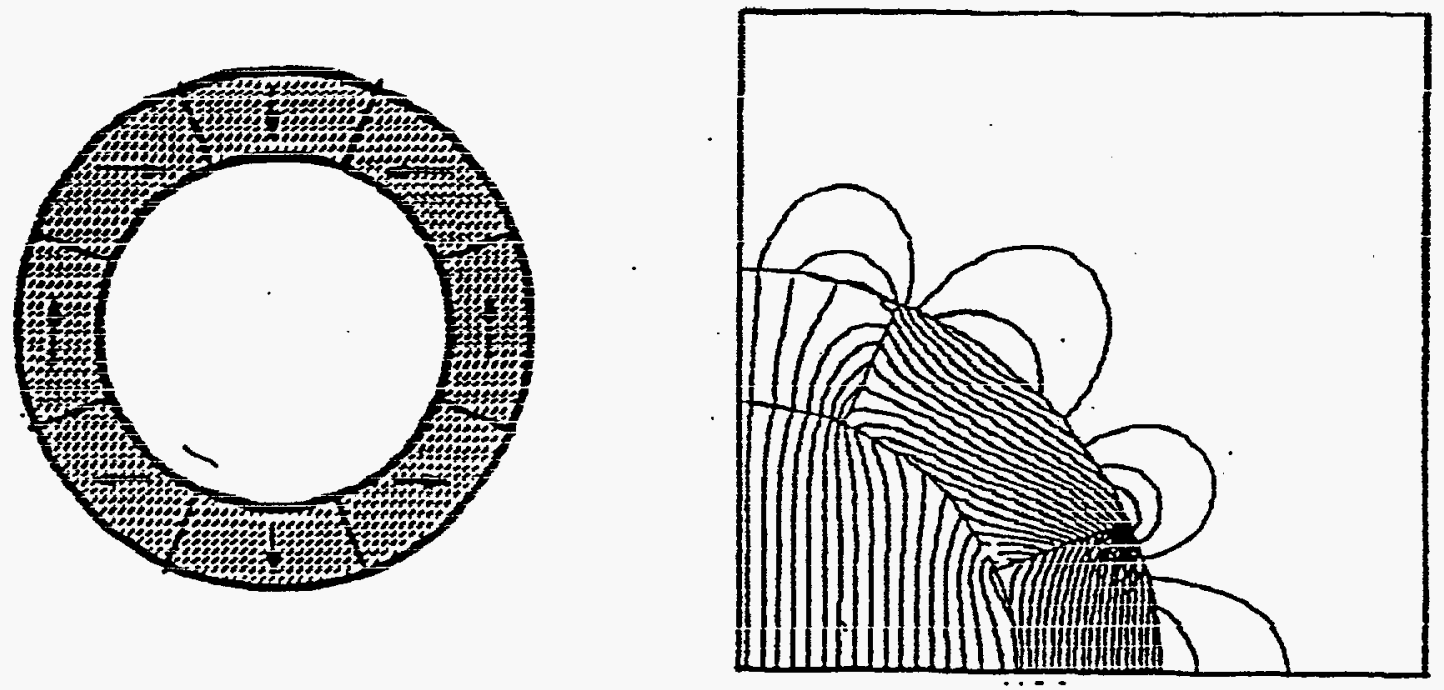

Figure 1. Cross Section and Field Lines of Halbach Array

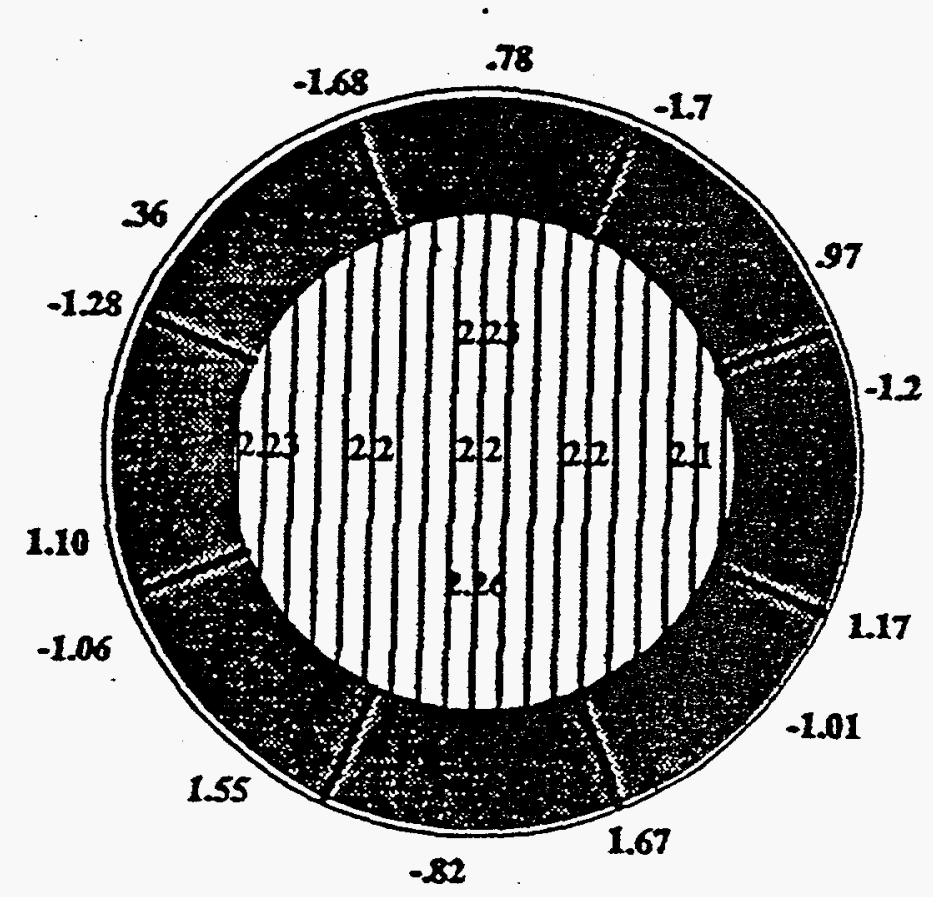

Figure 2. Measured Values of Halbach Array Field 


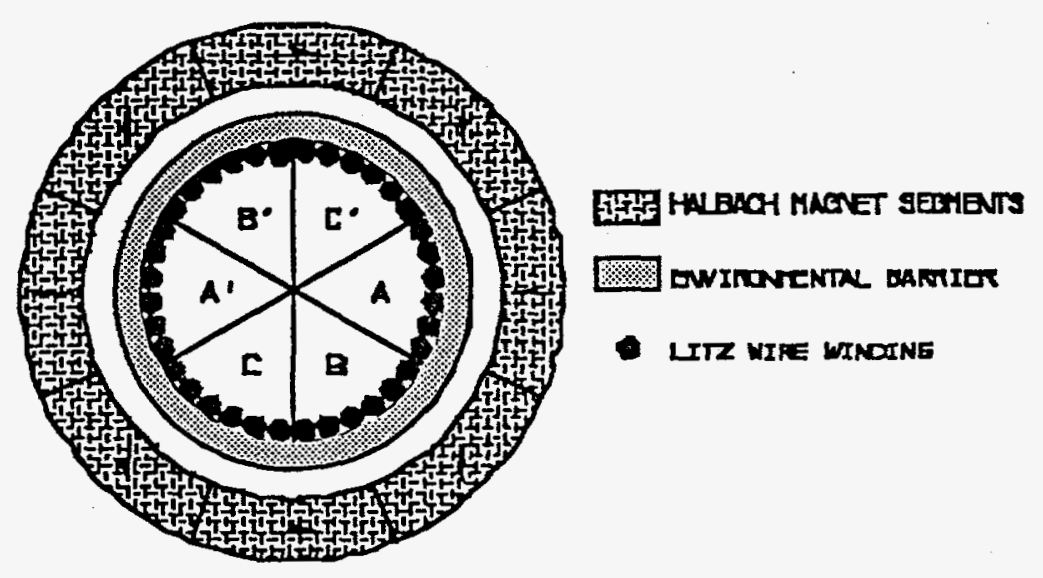

Figure 3. Halbach Array with Three Phase Winding

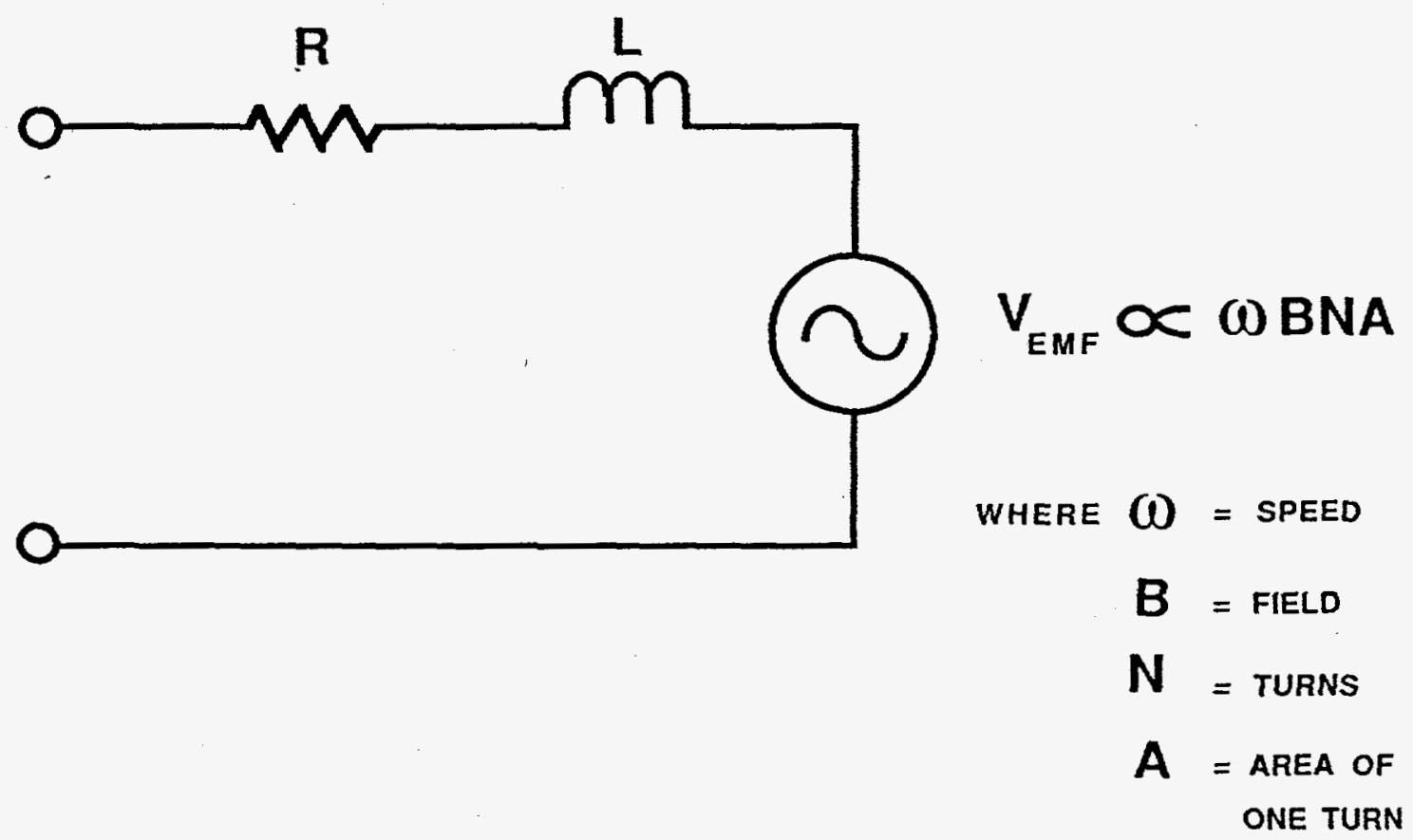

Figure 4. Equivalent Circuit for Electric Machine, per Phase Basis 


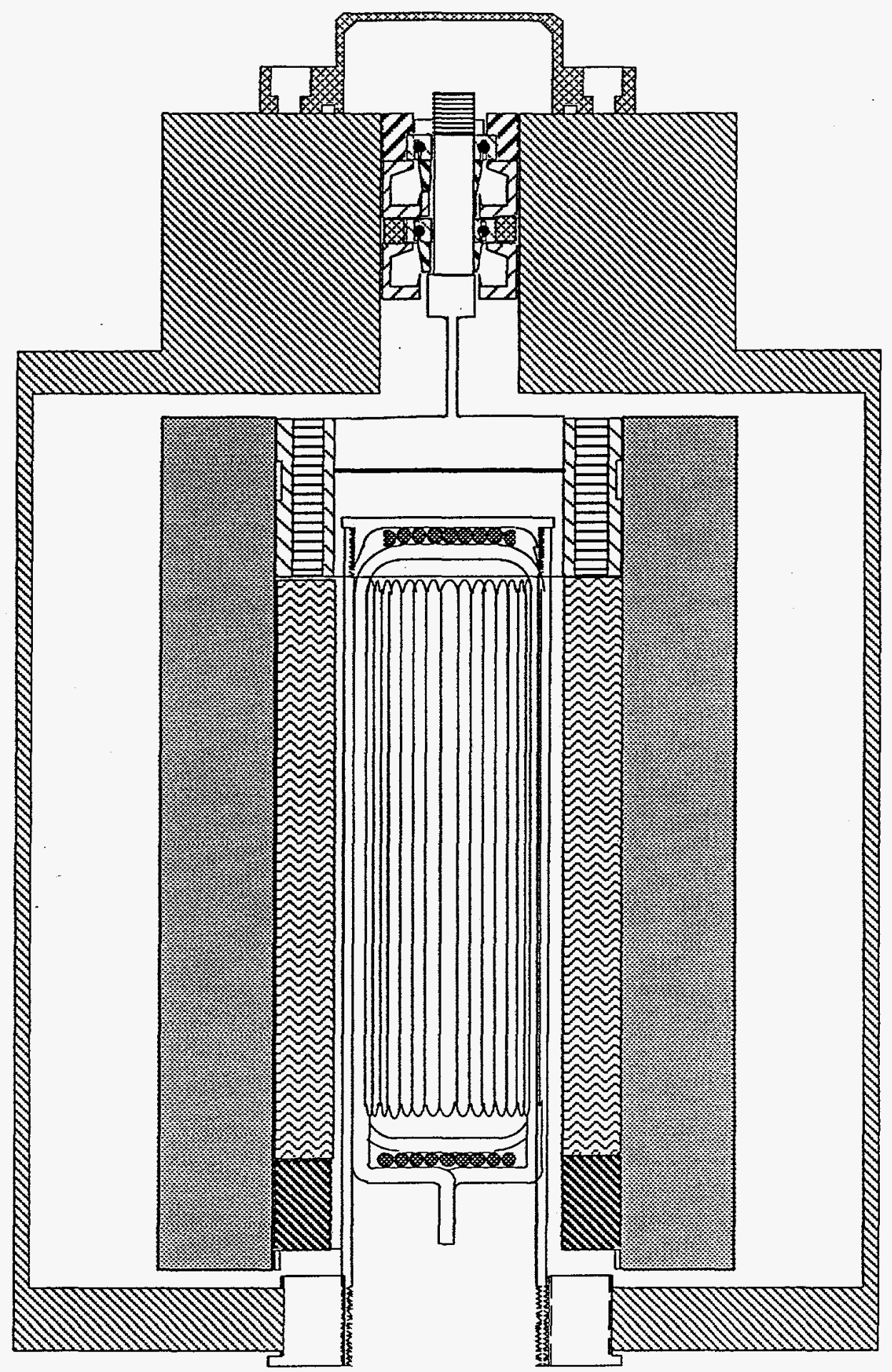

Figure 5. Electromechanical Battery 


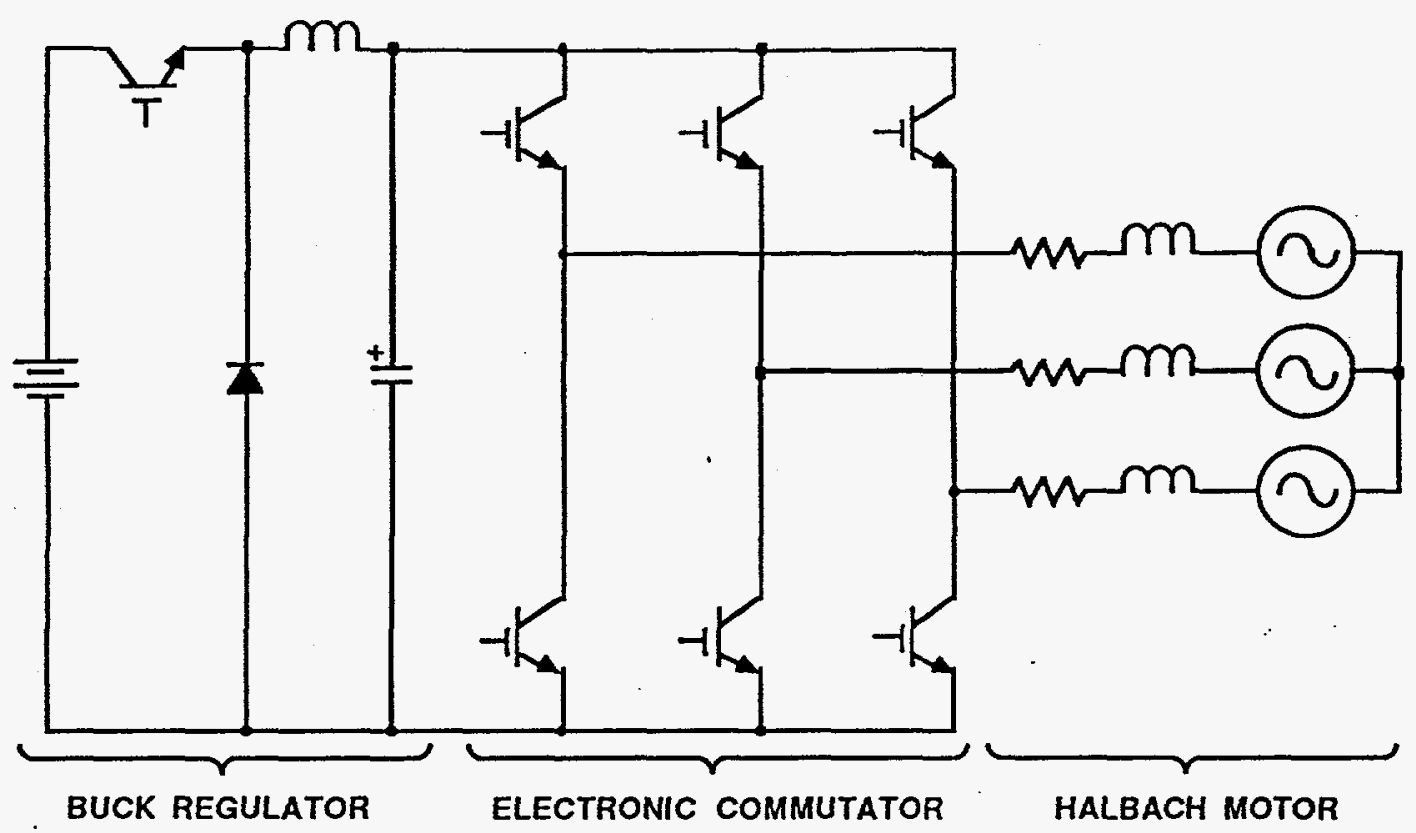

Figure 6. Simplified Schematic of Spin-up Circuit

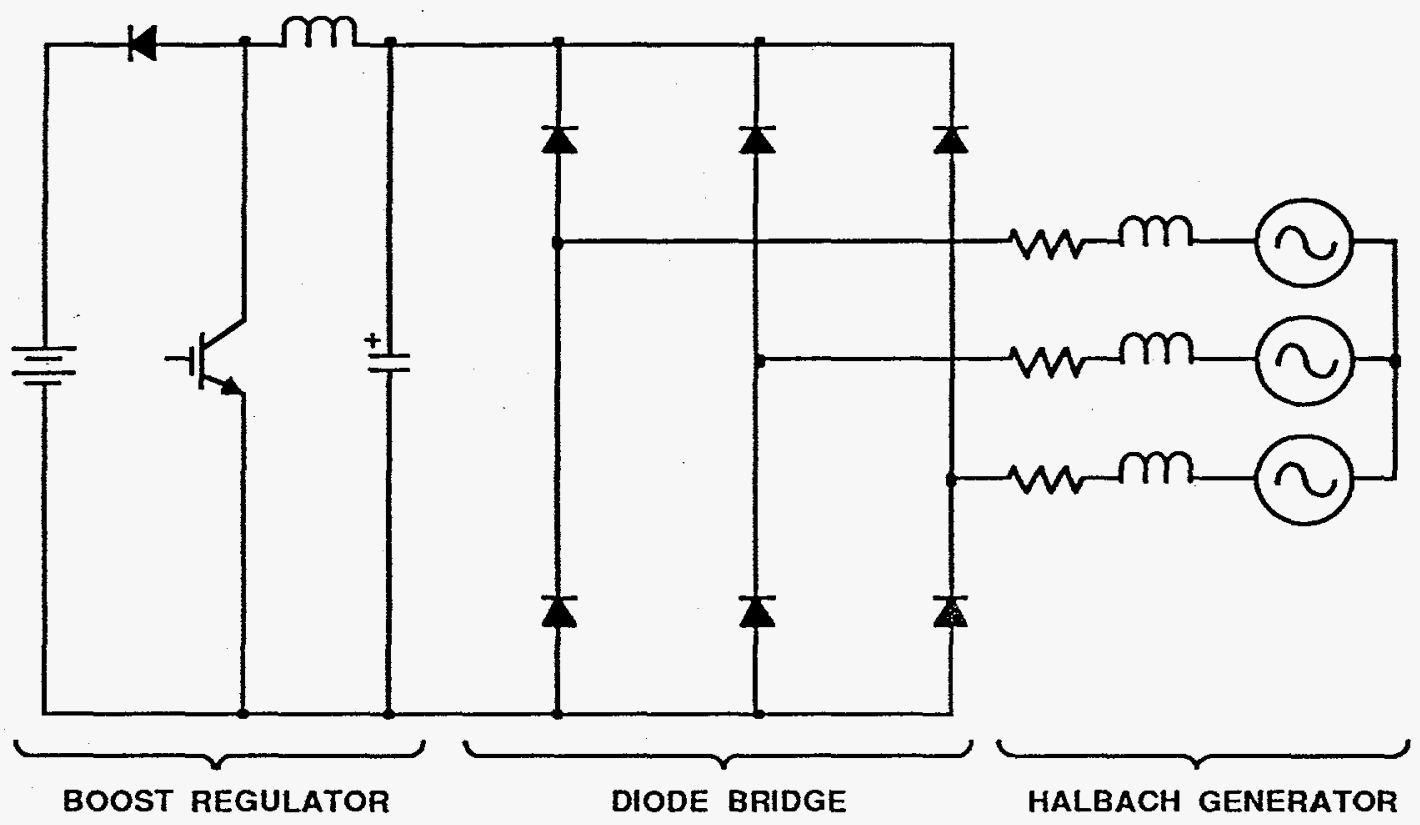

Figure 7. Simplified Schematic of Energy Extraction Circuit 


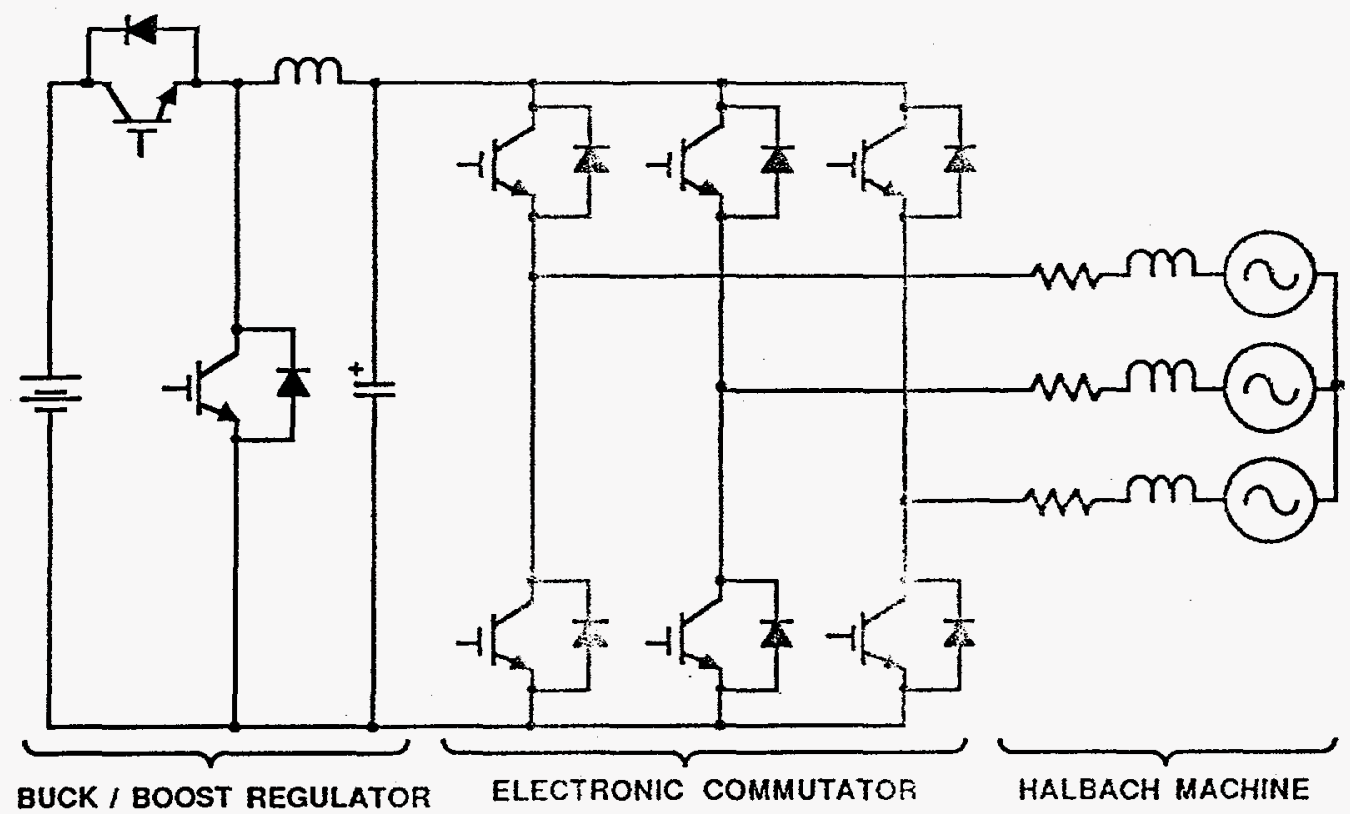

Figure 8. Simplified Schematic of Power Electronics for Electromechanical Battery

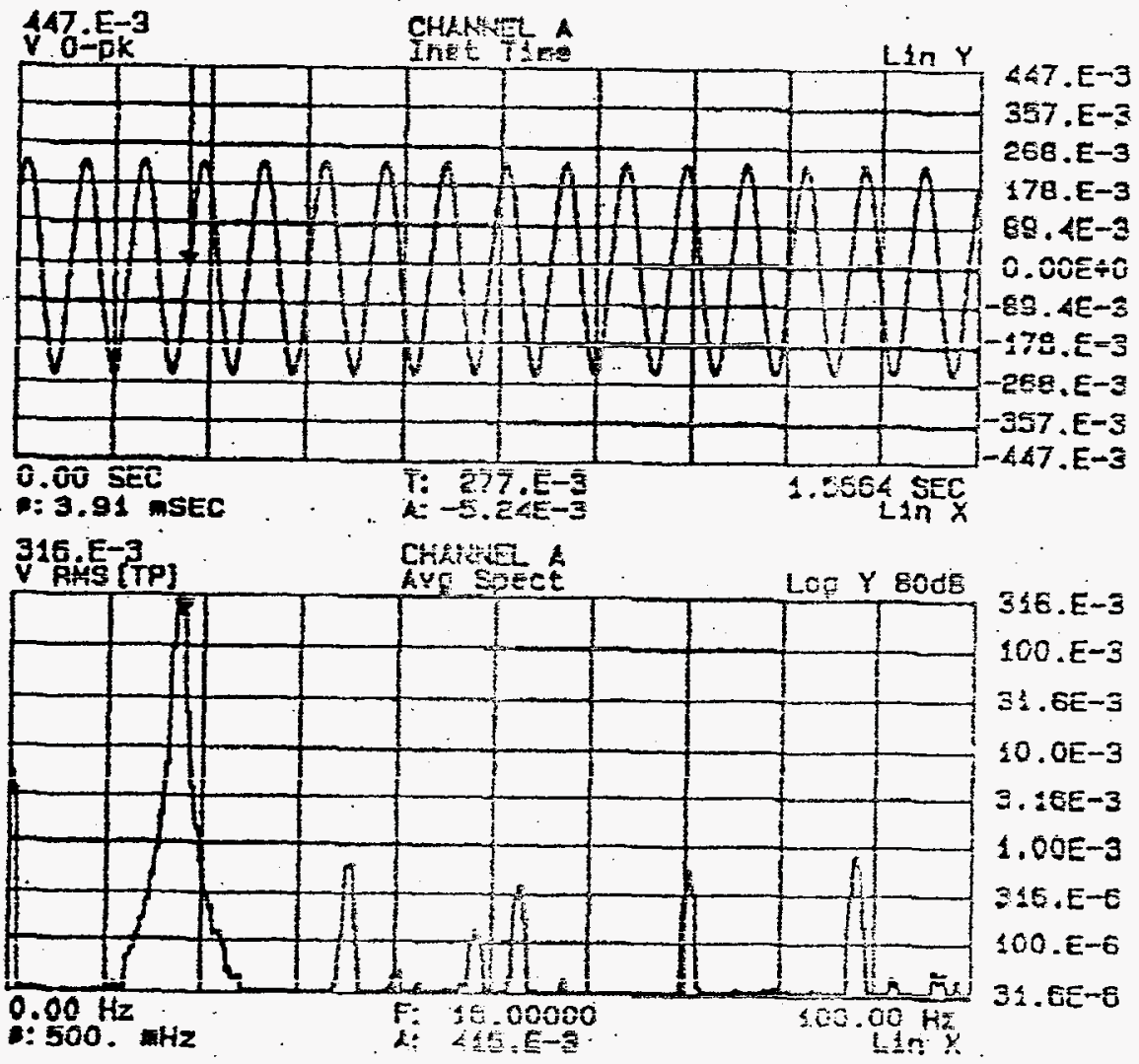

Figure 9. Open Circuit Voltage 


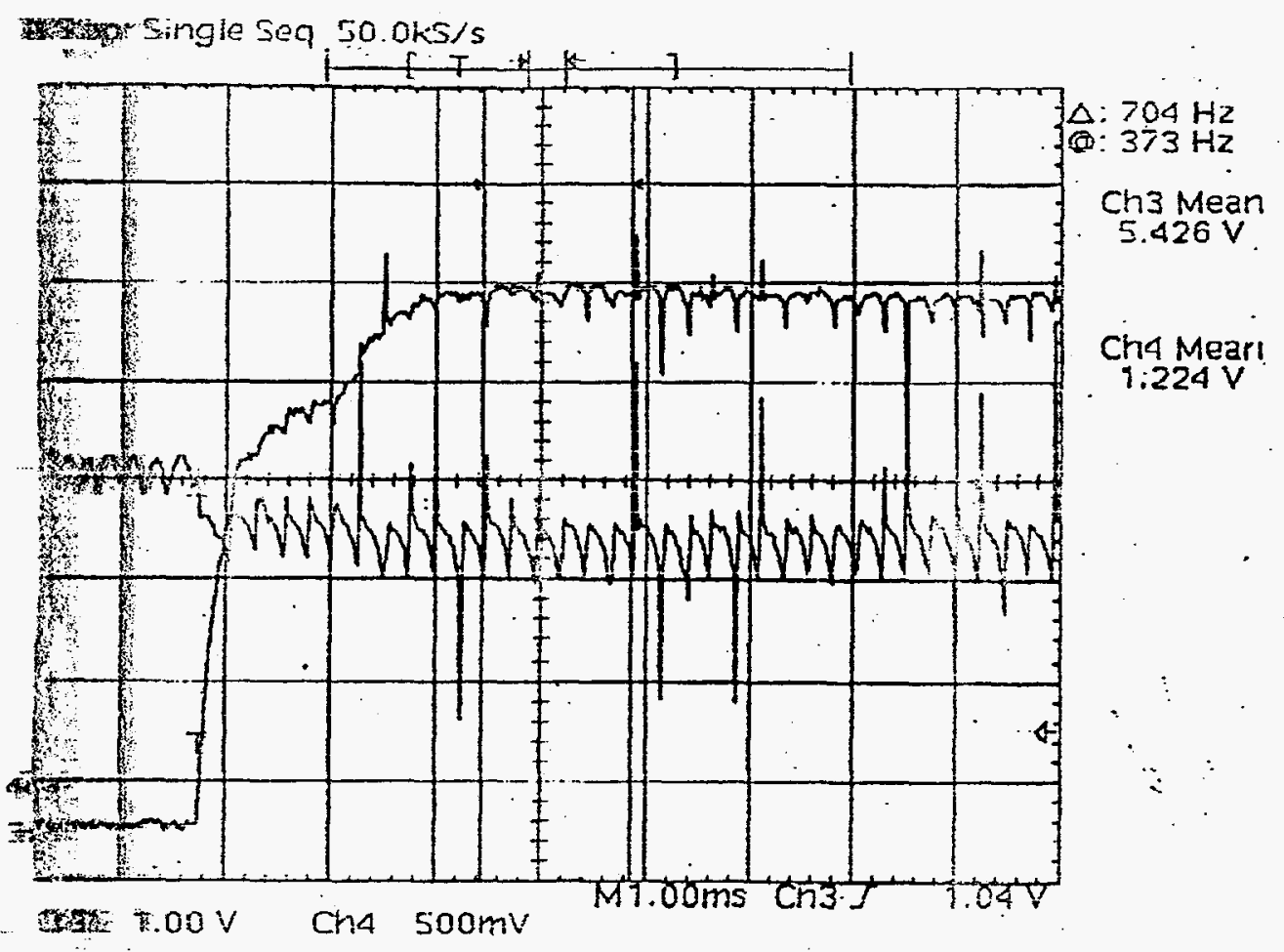

. Bridge Current and Voltage During Extraction, upper trace W0V/div, lower trace - current, 98A/div.

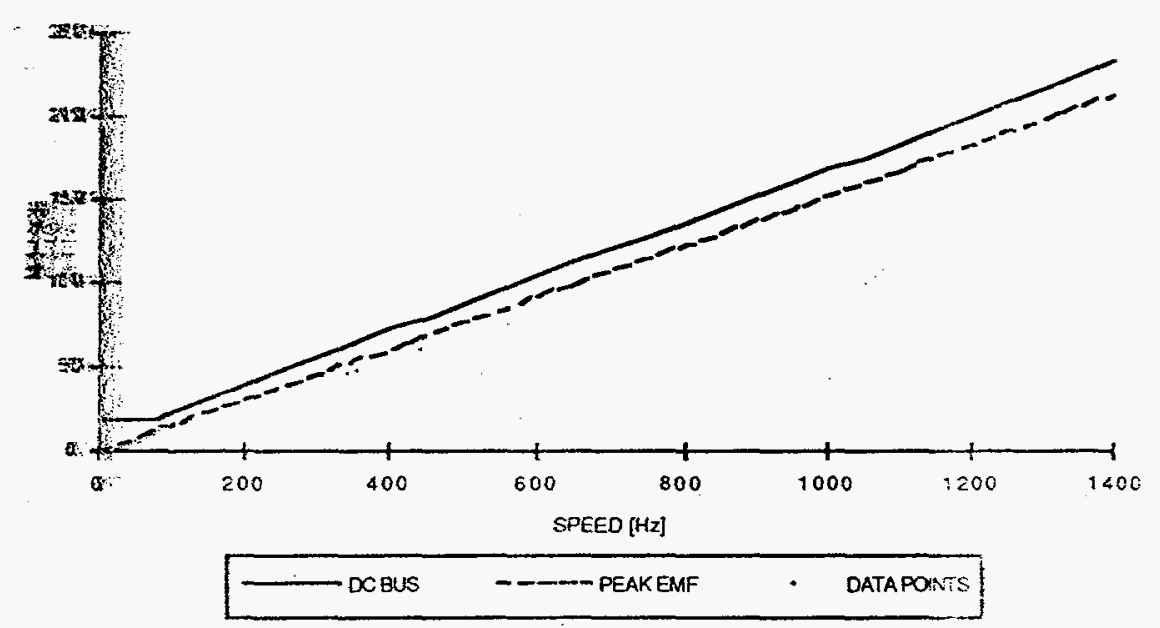

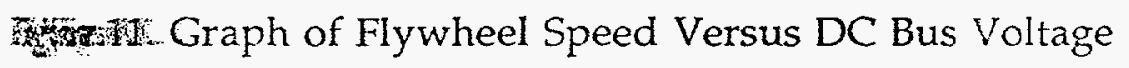

